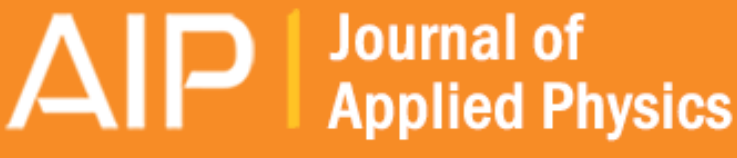

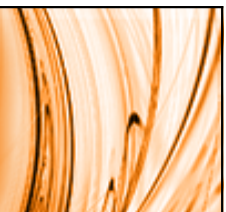

\section{Formation and magnetic manipulation of periodically aligned microchains in thin plastic membranes}

Daniela Lorenzo, Despina Fragouli, Giovanni Bertoni, Claudia Innocenti, George C. Anyfantis, P. Davide Cozzoli

, Roberto Cingolani, and Athanassia Athanassiou

Citation: Journal of Applied Physics 112, 083927 (2012); doi: 10.1063/1.4759328

View online: http://dx.doi.org/10.1063/1.4759328

View Table of Contents: http://scitation.aip.org/content/aip/journal/jap/112/8?ver=pdfcov

Published by the AIP Publishing

\section{Articles you may be interested in}

Study of polyvinyl alcohol nanofibrous membrane by electrospinning as a magnetic nanoparticle delivery approach

J. Appl. Phys. 115, 17B908 (2014); 10.1063/1.4867600

Relaxation of biofunctionalized magnetic nanoparticles in ultra-low magnetic fields

J. Appl. Phys. 113, 043911 (2013); 10.1063/1.4789009

Iron oxide nanoparticles fabricated by electric explosion of wire: focus on magnetic nanofluids

AIP Advances 2, 022154 (2012); 10.1063/1.4730405

A permalloy zigzag structure based magnetic bio-sensor

J. Appl. Phys. 111, 07E506 (2012); 10.1063/1.3676213

Micromagnet structures for magnetic positioning and alignment

J. Appl. Phys. 111, 07B312 (2012); 10.1063/1.3675067

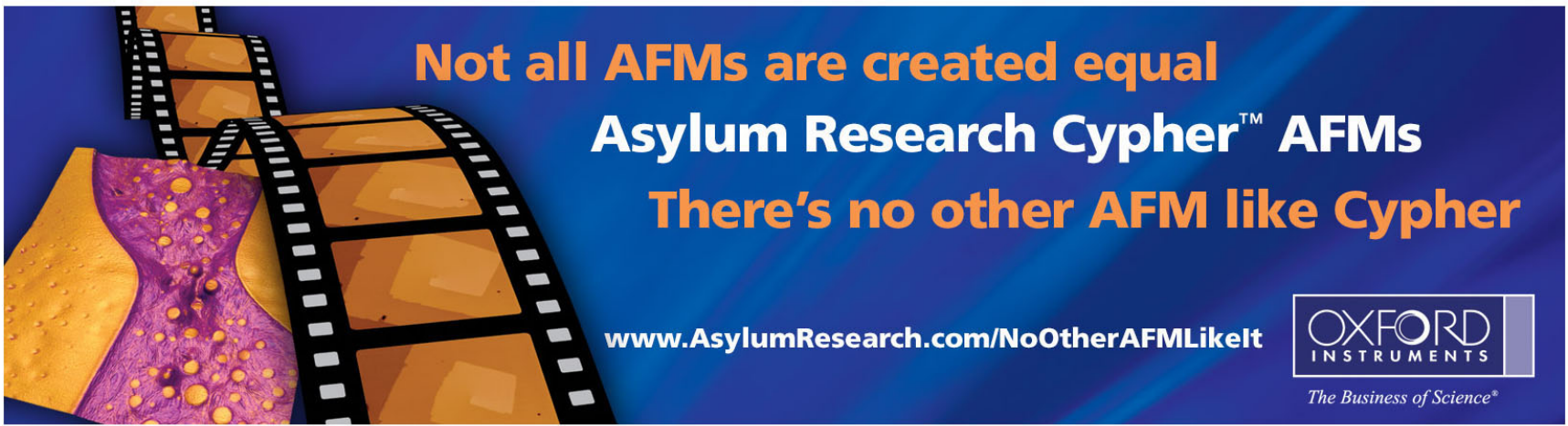




\title{
Formation and magnetic manipulation of periodically aligned microchains in thin plastic membranes
}

\author{
Daniela Lorenzo, ${ }^{1}$ Despina Fragouli, ${ }^{2, a)}$ Giovanni Bertoni, ${ }^{2,3}$ Claudia Innocenti, ${ }^{4}$ \\ George C. Anyfantis, ${ }^{1}$ P. Davide Cozzoli, ${ }^{5,6}$ Roberto Cingolani, ${ }^{7}$ \\ and Athanassia Athanassiou ${ }^{2}$ \\ ${ }^{1}$ Center for Biomolecular Nanotechnologies@UNILE, Istituto Italiano di Tecnologia, Via Barsanti, \\ 73010 Arnesano (LE), Italy \\ ${ }^{2}$ Nanophysics, Istituto Italiano di Tecnologia (IIT), Via Morego 30, 16163 Genova, Italy \\ ${ }^{3}$ IMEM-CNR, Parco Area delle Scienze 37/A, 43124 Parma, Italy \\ ${ }^{4}$ INSTM Research Unit and Dipartimento di Chimica, Universita“ di Firenze, via della Lastruccia 3, \\ 50019 Sesto F.no, Firenze, Italy \\ ${ }^{5}$ National Nanotechnology Laboratory (NNL), CNR Istituto Nanoscienze, clo Distretto Tecnologico, \\ via per Arnesano $\mathrm{km} \mathrm{5,73100} \mathrm{Lecce,} \mathrm{Italy}$ \\ ${ }^{6}$ Dipartimento di Matematica e Fisica "E. De Giorgi," Università del Salento, via per Arnesano, \\ 73100 Lecce, Italy \\ ${ }^{7}$ Istituto Italiano di Tecnologia (IIT), Via Morego 30, 16163 Genova, Italy
}

(Received 14 February 2012; accepted 25 September 2012; published online 26 October 2012)

\begin{abstract}
We demonstrate the fabrication of polymeric membranes that incorporate a few layers of periodically aligned magnetic microchains formed upon the application of variable magnetic fields. A homogeneous solution containing an elastomeric polymer and a small amount of colloidal magnetic nanoparticles is spin coated on glass slides, thereby forming thin magnetic membranes of ca. $10 \mu \mathrm{m}$ thickness. Subsequent application of a homogeneous magnetic field results in the orientation of the magnetic clusters and their further motion into the matrix along the field lines forming layers of aligned chains. The study of the kinetics of alignment demonstrates that the chains are formed in the first hour of exposure to the magnetic field. Above all, a detailed microscopy study reveals that the dimensions and the periodicity of the microchains are effectively controlled by the intensity of the magnetic field, in good agreement with the theoretical simulations. This ability to form and manipulate the size and the distribution of chains into the polymeric matrix gives the opportunity to develop multifunctional composite materials ready to be used in various applications such as electromagnetic shielding, or multifunctional magnetic membranes etc. (c) 2012 American Institute of Physics. [http://dx.doi.org/10.1063/1.4759328]
\end{abstract}

\section{INTRODUCTION}

Magnetic elastomers can be produced combining effectively a highly elastic polymeric matrix with magnetic nano/ micro fillers homogeneously dispersed therein. ${ }^{1}$ This combination results in hybrid magnetic materials with novel and often enhanced properties with respect to those of the individual components. Such nanocomposite materials can be used as display devices, ${ }^{2}$ magnetic actuators, ${ }^{3-6}$ magnetically controlled MEMS, ${ }^{7,8}$ microfluidic mixers, ${ }^{9}$ vibration absorbers, ${ }^{10}$ etc. On the top, the fabrication of magneto-elastomers incorporating magnetic periodic structures, such as aligned magnetic chains, results in anisotropic materials, where the physical characteristics of the matrix are effectively combined with the anisotropic properties of the relevant aligned structures. ${ }^{11}$ The resulting nanocomposites may present mechanical, ${ }^{12-15}$ magnetic, ${ }^{3,16,17}$ or optical anisotropy, ${ }^{18}$ which can find various applications, such as in the development of alternative optical devices ${ }^{19}$ or electromagnetic shields. ${ }^{20}$ Studies focused on this topic have demonstrated novel fabrication processes to pattern micro/nanostructures with controlla-

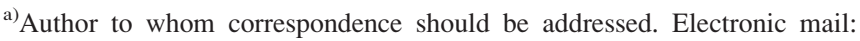
despina.fragouli@iit.it.
}

ble period using field-induced self assembly of nanoparticles (NPs) mainly in ferrofluids. ${ }^{21-24}$ In particular, columns consisting of aggregated structures of magnetic NPs have been developed, while numerous experimental and theoretical studies have been focused on the control of the distance between the columns as a function of the applied magnetic field. ${ }^{25-29}$ In all cases, the ferrofluids are placed in a defined cell, with the resulting columns having length determined by the cell thickness. This procedure results in periodic structures with defined length, aligned vertical to the samples surface, with non-permanent orientation, since when the magnetic field is removed the structures tend to disorient in their non rigid surrounding environment.

Here, we present the formation of stable horizontal chains, formed by superparamagnetic NPs in an elastomeric matrix due to the application of an external magnetic field $\left(\mathrm{B}_{0}\right)$ before and during the polymer curing, immobilized in a well-defined arrangement. In this way, elastomeric thin membranes containing few layers of aligned magnetic chains in the direction parallel to the plane of the membrane have been formed. A study on the variation of the dimensions of the chains and of the distance between them as a function of the $\mathrm{B}_{0}$ has been carried out. The experimental results are in good agreement with the theoretical study conducted. The ability to manipulate and 
fully control the dimensions and spatial arrangement of the chains in a horizontal plane offers significant prospects for the improvement of the properties of such composite materials, and their use in various applications.

\section{EXPERIMENTAL AND THEORETICAL METHODS}

\section{A. Samples preparation}

To prepare magnetic field-responsive polydimethylsiloxane (PDMS) composites, nearly spherical colloidal NPs of spinel-cubic $\gamma-\mathrm{Fe}_{2} \mathrm{O}_{3} / \mathrm{Fe}_{3} \mathrm{O}_{4}$ were used, synthesized by a modified surfactant-assisted nonaqueous synthetic approach, ${ }^{30,31}$ as described elesewhere. ${ }^{32}$ The monodispersed NPs with diameter $10 \pm 1 \mathrm{~nm}$ were prepared and solubilised in toluene at a concentration of $101.89 \mathrm{mM}$ (expressed in terms of $\mathrm{Fe}_{2} \mathrm{O}_{3}$ molecular units). PDMS (Sylgard 184 Silicone Elastomer) purchased from Dow Corning Corporation was supplied in two compounds: a pre-polymer and a crosslinker. Typical mixing ratio of pre-polymer to crosslinker is 10:1. All solvents used were purchased from Sigma Aldrich.

The PDMS pre-polymer was first dissolved in toluene, at a 1:1 weight ratio to achieve a diluted polymer base solution. Subsequently, it was mixed with the appropriate amount of NP solution, in order to achieve $0.4 \mathrm{wt}$. \% of iron oxide in polymer. Then, the PDMS crosslinker was added in a 10:1 (PDMS pre-polymer: crosslinker) weight ratio and mixed. In order to obtain the nanocomposite membranes, the final solution was spin-coated on glass substrates $\left(1 \times 1 \mathrm{~cm}^{2}\right)$ in one spinning step (1000 rpm for $10 \mathrm{~s}$ ).

Each membrane supported by the glass substrate was immediately placed under an external magnetic field generated by an electromagnet (Laboratorio Elettrofisico Walker LDJ Scentifico) overnight under ambient conditions, with the sample surface parallel to the direction of the homogenous $B_{0}$. The magnetic field intensity was adjusted by varying the voltage of the electromagnet and the distance between the two poles so as to obtain values ranging from 0 to $300 \mathrm{mT}$ as measured by a Gauss meter (GM 07 Hirst Magnetic Instrument Ltd.). During the application of the $\mathrm{B}_{0}$, apart from the aligned microchains formation, also the curing process started taking place and therefore the viscosity of the polymer increased gradually. After alignment completion and magnetic field removal, the samples were subsequently cured by heating at $95^{\circ} \mathrm{C}$ for $4 \mathrm{~h}$, in order to obtain the final solidification and chains immobilization in the polymer matrix. In this way, thin nanocomposite membranes of ca. $10 \mu \mathrm{m}$ thickness were formed, which represent the $5 \mathrm{wt}$. \% of the weight of the whole sample (membrane and glass substrate).

\section{B. Samples characterization}

For the study of the kinetics of the chains formation, it was used a set-up of two permanent magnets (500 mT each) placed at a distance of $5 \mathrm{~cm}$, using a transparent base. A sample of dimensions $1 \times 1 \mathrm{~cm}^{2}$ was placed at the center of the base, where it was subjected to a homogeneous magnetic field $\mathrm{B}_{0}=160 \mathrm{mT}$. The whole set up was placed under an optical microscope (Carl Zeiss, Axio Scope A1) equipped with a digital camera and image processing software, to image the microchains' formation in real time.

The width of the chains as a function of the $\mathrm{B}_{0}$ was measured using a confocal microscope (Leica TCS-SP5 AOBS) in bright field mode, using a laser light source of $405 \mathrm{~nm}$, and an oil immersion objective of $63 \times$ with a numerical aperture 1.40. The optical resolution of this type of instrument is $\mathrm{R}=200 \mathrm{~nm}$. The average nearest-neighbor distance between microchains as a function of $\mathrm{B}_{0}$ was statistically measured by studying the optical images using fourier transform analysis. The average length of the chains was measured using ImageJ. ${ }^{33}$ These three studies were made on membranes generated by the electromagnet.

Magnetic measurements were performed by a Quantum Design Ltd. SQUID magnetometer. The magnetization curves were acquired at room temperature $(300 \mathrm{~K})$, starting from an instrumental magnetic field of $5 \mathrm{~T}$ down to zero. The temperature dependence of ZFC-FC magnetization was collected under a static field of $5 \mathrm{mT}$ after cooling the samples down to $5 \mathrm{~K}$ in a zero magnetic field (ZFC curve) or in the same probe field of $5 \mathrm{mT}$ (FC curve). The magnetic characterization of the membranes supported by the glass substrates was carried out by aligning the magnetometer field in the plane of the nanocomposite film, first parallel and then perpendicular to the direction of the magnetic chains. Where possible, data were corrected by the diamagnetic contribution of the polymer and the glass support, separately measured. In the most of cases, however, the contribution of the glass substrate, which was dominant for high magnetic fields, hampers a correct evaluation of the total magnetization.

\section{Theoretical calculations}

Simulations were carried out using a Monte Carlo NVT algorithm. The system consisted of $\mathrm{N}=400$ particles in a $2 \mathrm{D}$ box with nearest image convention boundary condition. In order to simulate a setup close to the experiment, we considered as particles pre-formed clusters of diameters $d=200 \mathrm{~nm}$ (10\% standard deviation in diameters distribution), each one made of $10 \mathrm{~nm}$ superparamagnetic $\mathrm{Fe}_{2} \mathrm{O}_{3} \mathrm{NP}$ single units (a sort of coarse-grained simplification). High area fraction and cluster concentration of 0.03 (i.e. 3\%) were used as inferred from the experimental images of the final membranes. Thus it was considered that a large amount of the solvent was evaporated, while the particles could still move in the fluid medium (rough approximation of the experiment). Simulations were conducted for $\mathrm{B}_{0}=50,150$, and $300 \mathrm{mT}$.

\section{RESULTS AND DISCUSSION}

For the formation of the nanocomposite membranes containing aligned microchains on glass substrates, after the spin coating of the composite solution, external magnetic fields of various intensities are applied during the solvent evaporation and the curing of the polymer matrix. The applied fields in this study, $\mathrm{B}_{0}$, are always lower than the field required for the saturation of the NPs, $B_{s}$. As shown in Fig. 1, the magnetization curve does not show a complete saturation even at high fields, as usually observed for small NPs because of their surface contribution to the 


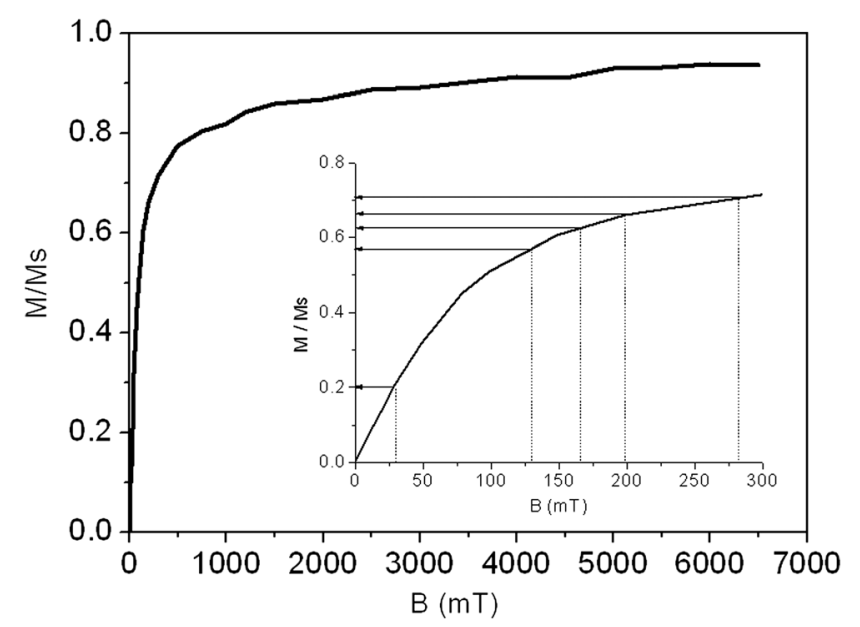

FIG. 1. Magnetization curve at $300 \mathrm{~K}$ for iron oxide NPs in toluene solution. Inset: details for low fields. Arrows denote the magnetization, normalized to the saturation magnetization, $\mathrm{M}_{\mathrm{S}}$, induced by fields corresponding to the $\mathrm{B}_{0}$ values used in this study $\left(B_{0}=30,130,160,200,280 \mathrm{mT}\right)$.

magnetization. For this reason, the $\mathrm{B}_{\mathrm{s}}$ can be defined as the field at which the magnetization reaches a high percentage (90\%) of the saturation value, $\mathrm{M}_{\mathrm{s}}$, evaluated by fitting the high field data to the empirical law: $M=M_{s}+a / B_{0}+b / B_{0}{ }^{2}$ $\left(\mathrm{B}_{\mathrm{s}} \approx 3 \mathrm{~T}\right)$. Analogously, the magnetization extent of the NP system induced by the external field can be estimated by the $\mathrm{M} / \mathrm{M}_{\mathrm{s}}$ ratio achieved for the corresponding $\mathrm{B}_{0}$ values, as shown in the inset of Fig. 1. It should be noticed that the $M_{s}$ of the NPs evaluated by considering the NP concentration in the toluene solution $(101.83 \mathrm{mM})$, is $62.6 \mathrm{~A} \mathrm{~m}^{2} / \mathrm{kg}$.

As shown in Fig. 2(a), the membrane formed without the field application presents inhomogeneous island-like structures, randomly dispersed in the polymer matrix, of diameter ranging from ca. 0.9 to $1.4 \mu \mathrm{m}$, most likely due to the interaction between single magnetic NPs with the polymeric macromolecules. ${ }^{34}$ By applying a uniform magnetic field parallel to the substrate during the polymer curing, the morphology of the membrane is noticeably changed (Fig. 2(b)). In particular, the isolated clusters, instead of aggregating in a random way, assemble into chain-like structures parallel to $\mathrm{B}_{0}$, circumventing the lateral assembly, and forming chains of various micrometers length.

In Figures 3(a) and 3(b), it is shown the representative ZFC/FC magnetization curves of the magnetic membranes prepared without (Fig. 3(a)) and with (Fig. 3(b)) the application of $\mathrm{B}_{0}$. The comparison to the NPs solution shows that the superparamagnetic behaviour of the system is not strongly modified by the polymer embedding procedure, apart from a small increase of the blocking temperature, $\mathrm{T}_{\mathrm{B}}$, denoting a higher inter-particle interaction in the polymer samples, due to the cluster or chain formation. The $T_{B}$ value, indeed, increases from $75.5 \mathrm{~K}$ for the solution sample to 84.4 $\mathrm{K}$ for the membrane including not-oriented NPs while a further increase of about $5 \mathrm{~K}$ is observed for the films prepared under the external field application. In addition, the magnetization dependence on the chain orientation is clearly evidenced by measuring the film oriented parallel and perpendicular to the magnetometer magnetic field (Fig. 3(b)), in accordance with our previous study, ${ }^{16}$ although due to the poor signal to noise ratio, no clear dependence on $\mathrm{B}_{0}$ can be appreciated among the different samples (supplemental material, Fig. S1). ${ }^{41}$ The room temperature magnetization curves of the same sample confirm the abovementioned results. In fact, in Fig. 3(c), the comparison with the data obtained for the iron oxide NPs measured in the toluene solution multiplied by their concentration in the polymer ( 0.4 wt. \%) proves that the magnetic properties of the pristine NPs are well maintained in the polymeric membrane. As expected, no coercivity is observed at room temperature, while the magnetization values are consistent with those expected considering the NP percentage embedded in the membrane. The small differences for the highest fields here reported can be ascribed to the error on the diamagnetic contribution evaluation of the glass substrate, which was dominant and it hampered a correct determination of the magnetization for higher fields. The magnetization curves in the range reported in Fig. 3(c), however, allow us to show the main difference due to the chains orientations, which is expected to decrease with increasing fields. ${ }^{16}$ In fact, as shown in the inset of Fig. 3(c), the effect of the membrane orientation is the lowering of the initial magnetic susceptibility when the chains are oriented perpendicularly to the magnetization field, as previously reported. ${ }^{16}$

Figure 4 presents the time evolution of the microchains formation under $\mathrm{B}_{0}$ of $160 \mathrm{mT}$. Specifically, immediately after placing the membranes under the external field, the induced magnetic moment of the superparamagnetic clusters is preferentially aligned parallel to the $\mathrm{B}_{0}$ direction, and the developed field gradients apply forces on the surrounding clusters resulting in their aggregation parallel to this direction forming elongated structures. Specifically, these structures move rapidly during the first minutes under the field, assembling with each other in a head-to-tail direction (see video of supplemental material). ${ }^{41}$ Progressive addition of

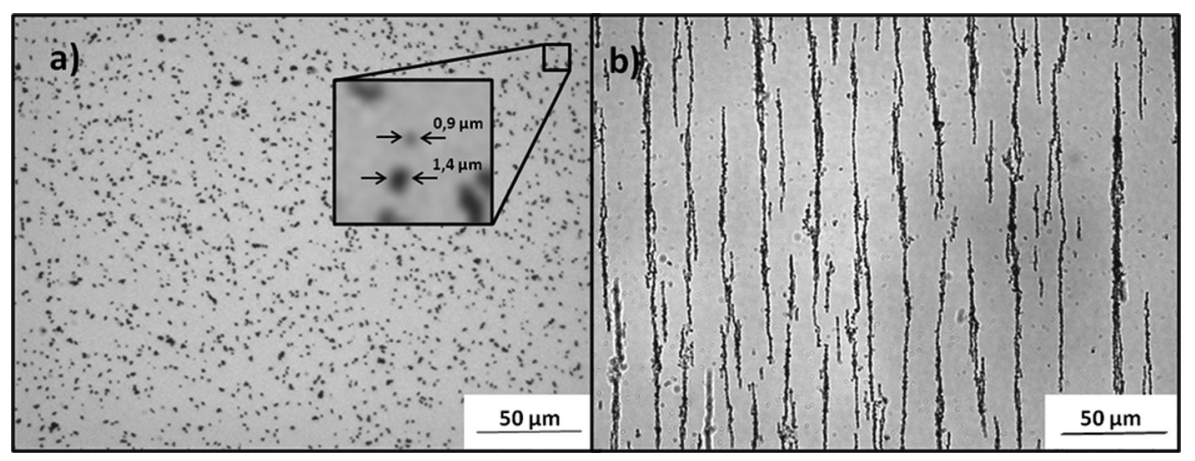

FIG. 2. Optical microscopy images of (a) a film formed without magnetic field application and (b) a film formed under an $\mathrm{B}_{0}$ of $130 \mathrm{mT}$. 


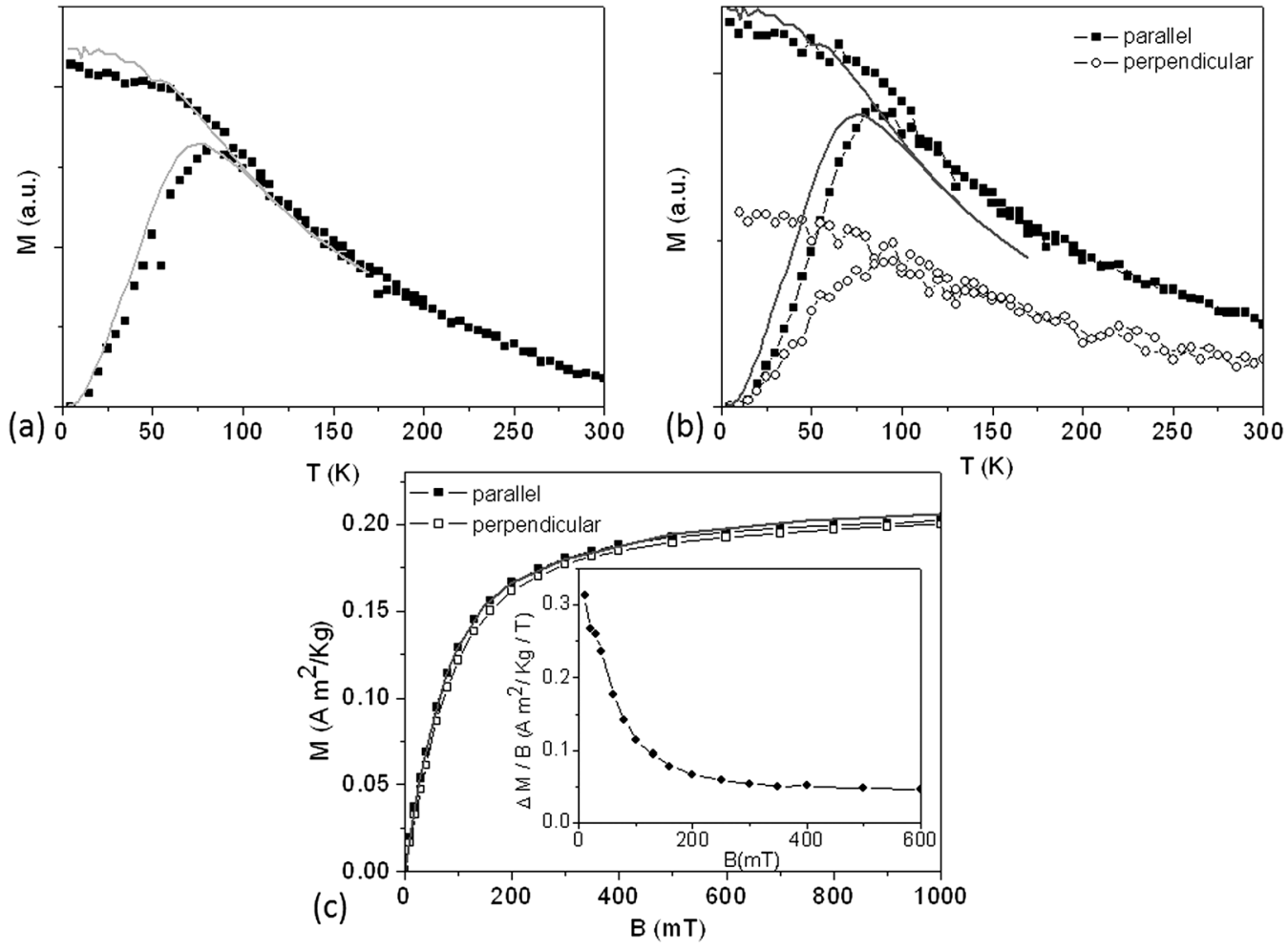

FIG. 3. (a),(b) ZFC/FC magnetization curves collected for a film formed without magnetic field application and under $\mathrm{B}_{0} 130 \mathrm{mT}$, respectively. In this latter case, the measurement was preformed twice by aligning the magnetic chains parallel and perpendicular to the instrumental magnetic field. The continuous line represents the ZFC/FC magnetization curves acquired for NPs dispersed in toluene solution. The acquisition was stopped at $180 \mathrm{~K}$ to prevent the defreezing of the solution. (c) Magnetization curves for the same film collected at $300 \mathrm{~K}$. Magnetization values are normalized to the weight of the membrane. Error bars are included in the symbol size. The continuous line represents the magnetization curve obtained for the NPs in toluene solution multiplied by the estimated percentage contained in the membrane. In the inset, the magnetization difference between the two orientations divided by the application field is reported.

neighboring clusters to the edges of the early formed elongated structures causes a linear increase of the length of the chains, approaching $80 \mu \mathrm{m}$ after ca. $60 \mathrm{~min}$ (Fig. 5), and the concomitant reduction of randomly oriented clusters. A closer look reveals that neighboring clusters move not only parallel to the $\mathrm{B}_{0}\left(\theta=0^{\circ}\right)$ but also in other directions $\left(0^{\circ}<\theta<90^{\circ}\right)$ in order to join the edges of the closest microchains (head or tail) within a distance of ca. $11.5 \mu \mathrm{m}$. In fact, as the newly formed chains increase in length, their collective dipole moment increases, and consequently their induced field gradients affect also other small chains and clusters at larger angles, attracting them in the head-to-tail direction. For longer distances (typical of diluted solutions), NP clusters tend to orient individually forming new chains, which are far away from their neighbors. At the end of the process long and stable microchains are formed, possibly with few clusters dispersed between them, distributed at a minimum possible distance in a periodic way since this is the optimal arrangement for their internal magnetic fields to be in a dynamic equilibrium. ${ }^{35}$

Once the dynamics of the mechanism of the microchains' formation is clarified, we discuss how the width and length of the final, definitive microchains, and their average relative distance depend on the $\mathrm{B}_{0}$. Figure 6 demonstrates the optical microscopy images of different membranes obtained at three different $\mathrm{B}_{0}(30,130$, and $200 \mathrm{mT})$. In particular, it is shown that the chains formed upon application of $\mathrm{B}_{0}=30 \mathrm{mT}$ look shorter, thicker, and are more distant compared to the chains obtained at higher intensities. As the $B_{0}$ increases, the length of the chains increases, while their diameter and lateral distance decrease. Particularly, for $\mathrm{B}_{0}$ values from 30 to $280 \mathrm{mT}$, the length of the formed chains increases from 40 to $255 \mu \mathrm{m}$, while their diameter decreases, starting from ca. $1.1 \mu \mathrm{m}$ for the minimum applied field and reaching a minimum value $0.6 \mu \mathrm{m}$ at the maximum value of $\mathrm{B}_{0}=280 \mathrm{mT}$. Plotting the ratio of the width of the chains to their length versus the external magnetic field intensity at Fig. 7(a), it can be seen that it decreases non linearly with the increase of $\mathrm{B}_{0}$. Moreover, as shown in Fig. 7(b), the spacing between the chains can be effectively tuned, since it decreases with the field, from $20 \mu \mathrm{m}$ to $9 \mu \mathrm{m}$. The same spacing between the chains is expected not only in the plane parallel to the surface of the membranes but also in the vertical one, e.g. throughout their thickness. Due to this fact and given that the thickness of the developed membranes is ca. $10 \mu \mathrm{m}$, it is estimated the formation of one or maximum a couple of layers of aligned chains parallel to the substrate, as also verified by the microscopy study. There are several theoretical models that explain this type of behavior, most of them being focused on the formation of ferrofluid patterns of defined length vertically assembled with respect to the substrate. ${ }^{36,37}$ All of them address the problem by minimizing the free energy of the system, by keeping the length of the chains or the thickness or the external magnetic field stable. Following this approach, the power law dependence of the periodic patterns on the external magnetic fields can be 

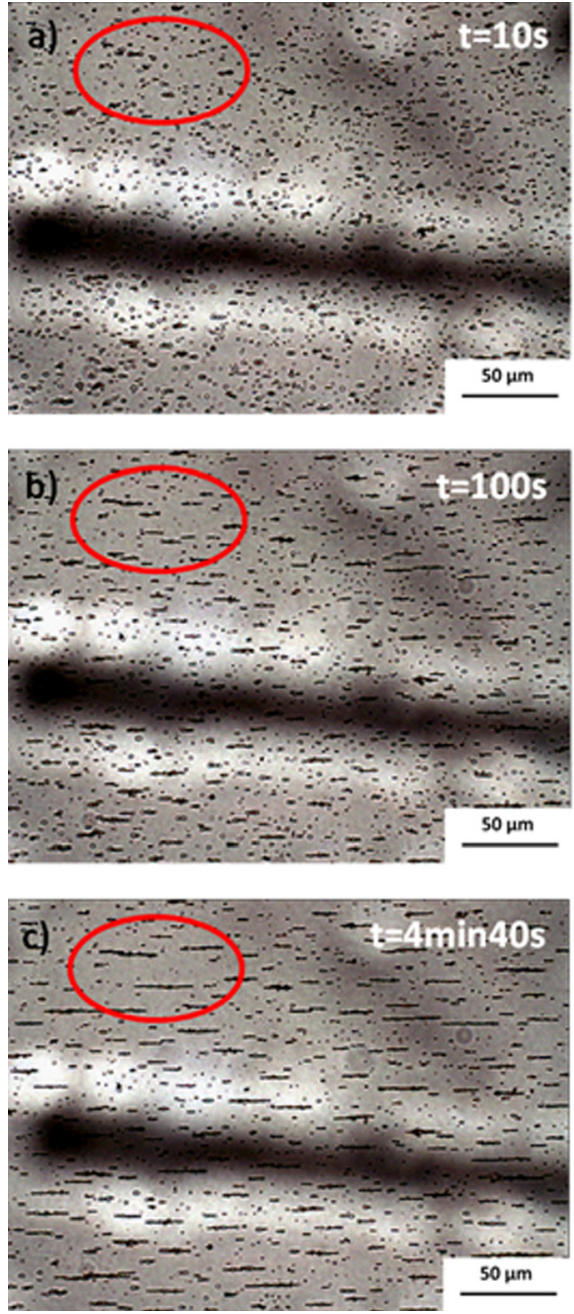
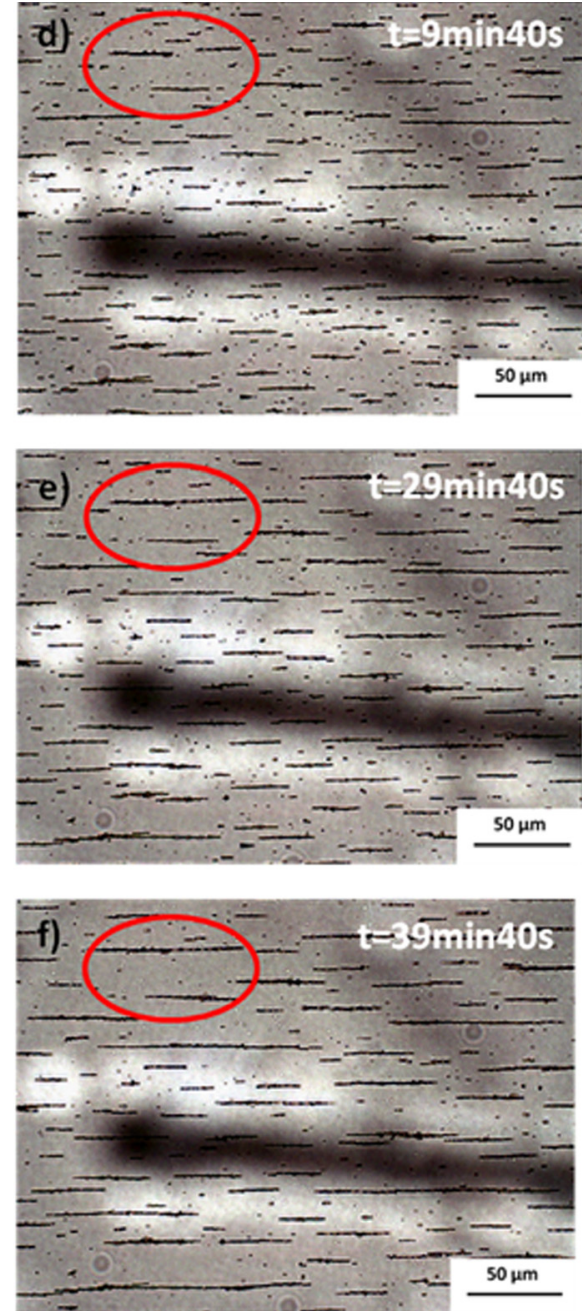

FIG. 4. Microscopy images of the alignment of the iron oxide clusters at different times: (a) $\mathrm{t}=10 \mathrm{~s}$, (b) $\mathrm{t}=1 \min 40 \mathrm{~s}$, (c) $\mathrm{t}=4 \min 40 \mathrm{~s}$, (d) $\mathrm{t}=9 \min 40 \mathrm{~s}$, (e) $\mathrm{t}=29 \min 40 \mathrm{~s}$, (f) $\mathrm{t}=39 \min 40 \mathrm{~s}$ under an external $\mathrm{B}_{0}=160 \mathrm{mT}$. derived, with the exponent values being negative, and close to -1 . The presented results are in agreement with these observations although all parameters are varying and moreover the external magnetic field is sufficiently larger compared to the other studies. Specifically, the way the ratio of the chains width/length, and the period of the chains are varying with the $\mathrm{B}_{0}$ is similar, as shown by the derived expo-

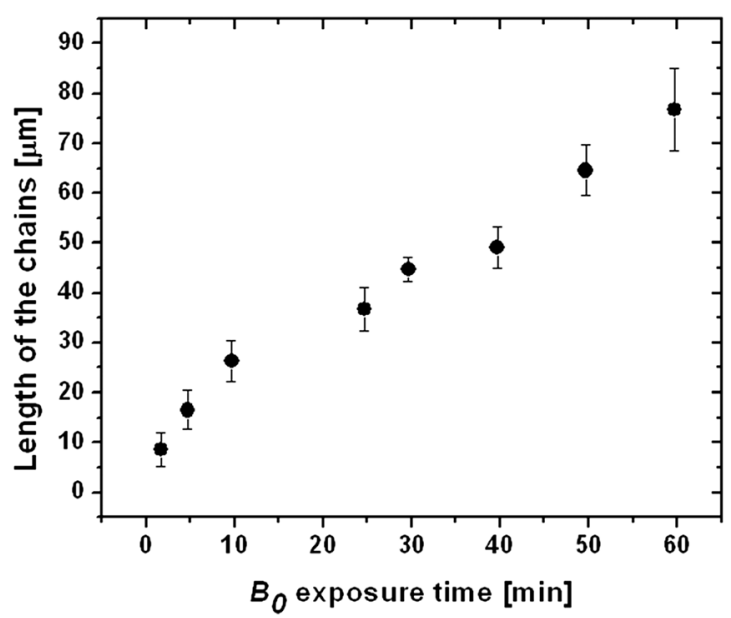

FIG. 5. Length of the microchains as a function of the exposure time to an external magnetic field of $\mathrm{B}_{0}=160 \mathrm{mT}$. nential factors in Figs. 7(a) and 7(b) $(-1.16 \pm 0.08$ and $-0.32 \pm 0.03$, respectively). Finally, the energy of interaction between clusters at low magnetic field intensities is not sufficient to move all the clusters to form periodic chains. For this reason, elongated structures of length between 1.5 and $10 \mu \mathrm{m}$ can be found dispersed in the film between longer periodic chains. However, with increasing $\mathrm{B}_{0}$, the number of these structures decreases, as demonstrated in Fig. 7(c), where it is plotted the normalized density (number of elongated structures of length $<5 \mu \mathrm{m}$ per unit area) as a function of the applied external field.

To explain the above described results of the effect of the $\mathrm{B}_{0}$ on the structural characteristics of the formed microchains into the elastomeric matrix we propose the following mechanism. Assuming that the initial clusters of NPs presented in Fig. 2(a) have sizes in the same range, the strength of the dipolar moment interactions between them increases with the intensity of the applied $\mathrm{B}_{0}$, as described by the coupling constant $\lambda_{0}$ (Eq. (1)), ${ }^{38}$

$$
\lambda_{0}=\frac{\mu_{0} m_{0}^{2}}{4 \pi d_{0}^{3} k_{B} T}=\frac{\mu_{0} N^{2} m_{p}^{2}}{4 \pi d_{0}^{3} k_{B} T} L^{2}\left(\frac{m_{p} B_{0}}{k_{B} T}\right)
$$

with $\mu_{0}$ the magnetic permeability in vacuum, $d_{0}$ the cluster radius, $m_{0}$ and $m_{\mathrm{p}}$ the magnetic moments of the clusters of particles and the single particles respectively, $L$ the Langevin 


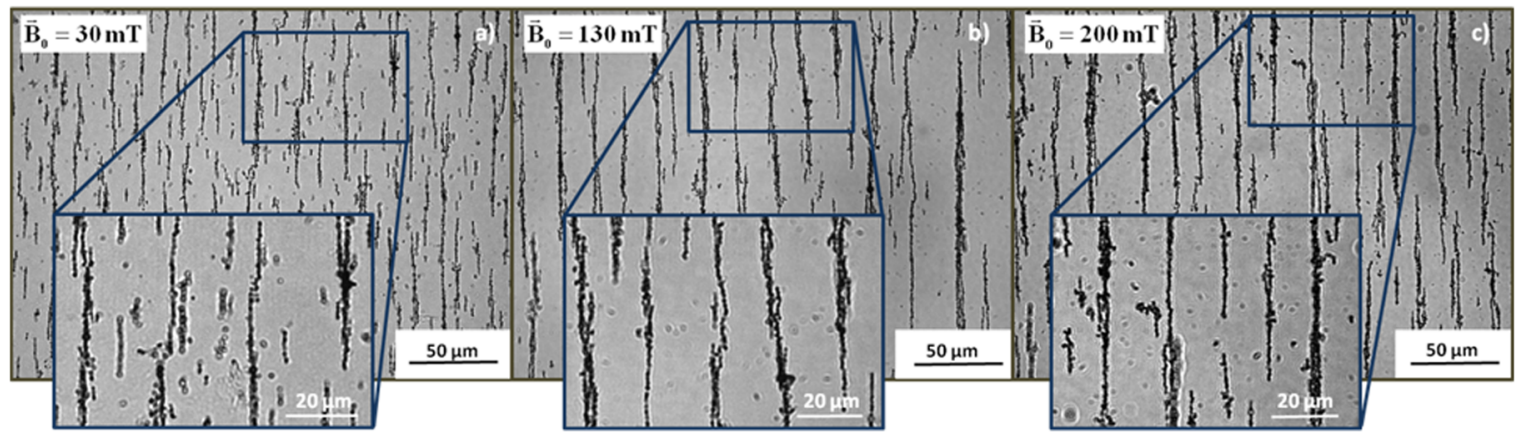

FIG. 6. Optical microscopy images of final films formed under magnetic fields with intensity (a) $\mathrm{B}_{0}=30 \mathrm{mT}$, (b) $\mathrm{B}_{0}=130 \mathrm{mT}$, (c) $\mathrm{B}_{0}=200 \mathrm{mT}$.

paramagnetic function corrected by the contribution of the NPs anisotropy, ${ }^{39} k_{\mathrm{B}}$ the Boltzmann constant, $T$ the temperature, and $N$ the number of superparamagnetic NPs forming each cluster. Equation (1) assumes that a cluster can be seen as a paramagnetic sum of the contributions of its constituent NPs. Thus, when the samples are exposed to the various external magnetic fields, in the very first seconds, the higher the $\mathrm{B}_{0}$ is, the more intense the interaction between clusters as shown by the coupling constant $\lambda_{0}$. Therefore, it can be safely assumed that in the case of a high $\mathrm{B}_{0}$, the clusters start to arrange and interact with neighbor clusters very fast, efficiently forming elongated structures. We can expect that an elongated structure $(i)$ has a higher induced magnetic moment compared to the magnetic moment of a small cluster (j). This interaction is maximum in the direction parallel to the dipoles direction, while as the angle between the magnetic moment and the induced magnetic field increases it becomes smaller $\left(U_{m}=-\vec{m}_{i} \cdot \vec{B}_{i j}\right)$. Thus, we expect that clusters are attracted by the elongated structures that are initially formed in their close vicinity, and rapidly assembled onto their heads or tails, forming eventually the microchains.
On the other hand, low $\mathrm{B}_{0}$ induces weak interactions between the clusters, resulting in a statistically lower number of initially formed elongated structures. This means that the magnetically strong centers which attract the clusters in head-to-tail configuration appear reduced compared to the structures formed under strong magnetic fields. Thus, the interaction of the clusters may be done also laterally resulting into thicker chains with larger distances between them. Furthermore, the partial curing of the polymer even at room temperature and the evaporation of the remaining solvent during this procedure, changes also the viscosity of the matrix, thus there is a decreased possibility to form longer chains with increasing time. For this reason the longer and thinner chains appear in the membranes formed under high magnetic fields, since they are formed faster.

In order to perform the Monte Carlo NVT simulations we took into account that the particles initially aggregate into clusters. In particular, the simulations were performed using a cluster moving algorithm as explained in Refs. 38 and 40, and considering bigger particles (clusters) of $\mathrm{d}=200 \mathrm{~nm}$ diameter. Each cluster was considered as a single particle, with a
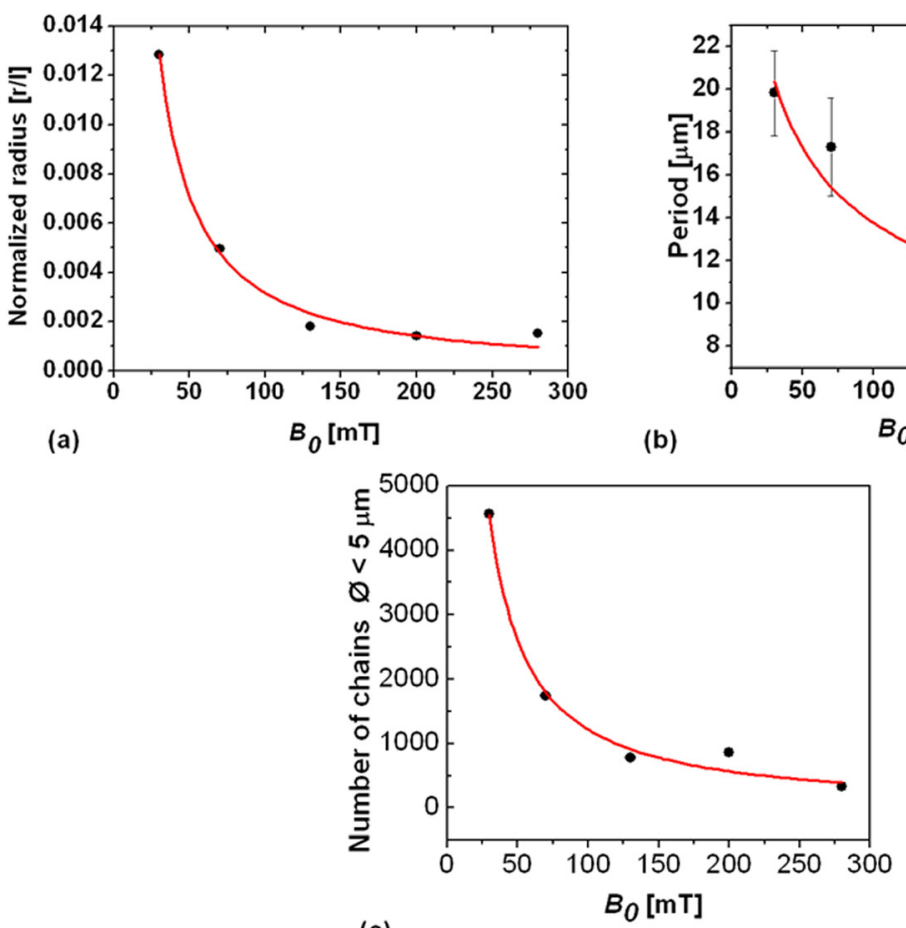

FIG. 7. (a) Average radius/length dependence of the chains on the external magnetic field $B_{0}$. (b) Average period of the chains versus $B_{0}$. (c) Normalized density (number of elongated structures of length $<5 \mu$ m per unit area) as a function of $\mathrm{B}_{0}$. The red curves show the power law dependence derived by the fitting curve $\mathrm{y}=\mathrm{A} \mathrm{x}^{\mathrm{b}}$ with $\mathrm{b}=-1.16 \pm 0.08$ for (a) $\mathrm{b}=-0.32 \pm 0.03$ for (b), and $b=-1.094 \pm 0.08$ for (c). 


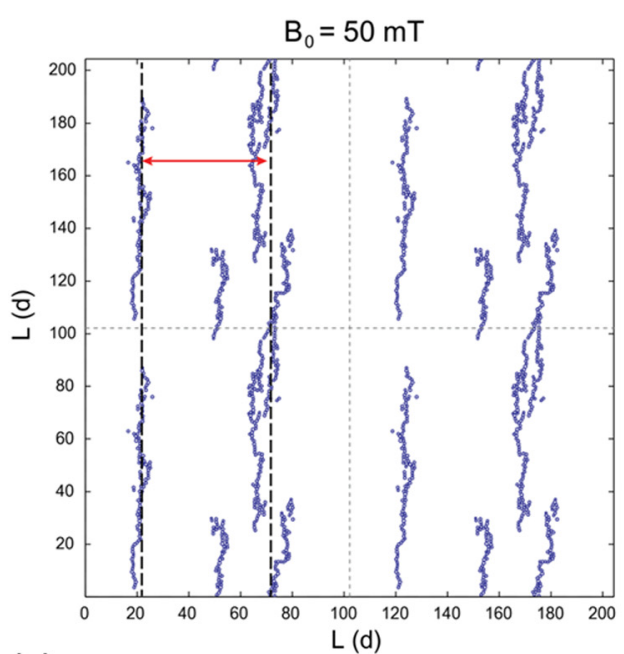

(a)

magnetic moment given by the paramagnetic superposition of the NP moments (see Eq. (1)), where the Langevin function is corrected by the contribution of the NPs anisotropy. As a result, the magnetic moments are much higher than in the case of single NPs, so that the van der Waals attractions are negligible. We considered in this way a net magnetization of the clusters due to the $\mathrm{B}_{0}$, to test whether the dipolar magnetic interactions can drive the alignment of the chains and also to verify the inter-chain distance dependence on the external magnetic field. As shown in Fig. 8, the chains result in being thinner and longer at $\mathrm{B}_{0}=300 \mathrm{mT}$. This reflects the favored configuration with dipoles pointing in the direction of the field and in a head-to-tail configuration. At low field magnitudes, as $50 \mathrm{mT}$, the chains (the simulation is limited to a 2D system) exhibit a more pronounced branched configuration, resulting in an effective larger occupied area.

The results are similar to the case of small particles ${ }^{40}$ at high fields. However, in that case at low magnetic fields $\left(\mathrm{B}_{0}=50 \mathrm{mT}\right)$, the formed structures in the final configuration resemble elongated structures rather than defined chains as observed in the experiment (supplemental material Fig. S2). ${ }^{41}$ On the contrary, the higher magnetic moments of the clusters give rise to better defined chains also at low external magnetic fields $\left(\mathrm{B}_{0}=50 \mathrm{mT}\right)$. As for the case of small particles, the chains at $\mathrm{B}_{0}=300 \mathrm{mT}$ are thinner and straighter, and result at closer distances. We can think of the balance between dipolar interactions in this way: thicker chains stay at higher distance, thin chains at closer distance. Moreover, it is clear that at $300 \mathrm{mT}$, the chains are longer than at $50 \mathrm{mT}$. The similar behavior for NPs and clusters of NPs indicates that the results can be scaled up to the actual experimental dimensions (clusters up to $500-1000 \mathrm{~nm}$ ).

Even if this model cannot catch the dynamics of formation of the chains or provide the mechanism of the detailed process, ${ }^{40}$ it indicates that the clustering of the NPs as initial step is fundamental in the chains formation. The clusters move as single units under the applied field, favoring the chain formation also in the case of very small particles (as the $10 \mathrm{~nm}$ NPs of the experiment). The lengthening and thinning of the chains together with the decrease of the intra chains distance with the applied filed are confirmed by the simulation. Possibly improvements to the model concern the use of more particles in a bigger box, to reduce the effect of the boundary conditions, or including detailed contributions from the particles at the surfaces of the clusters. Both tasks however imply a large increase of computational time.

\section{CONCLUSIONS}

Patterned microstructures with controllable length, width, and periodicity can be achieved using iron oxide NPs aggregated into chain-like structures within elastomeric polymer films upon the application of magnetic field parallel to the substrate. The pattern characteristics are controlled by the intensity of the applied magnetic field. Detailed experimental and theoretical studies demonstrate that the period of the aligned structures decreases as the magnetic field intensity increases, while the chains become longer and thinner. This is due to the dipolar interactions between the clusters, which directly depend on the strength of the external magnetic field.

\section{ACKNOWLEDGMENTS}

The authors acknowledge partial financial support by the Italian Ministry of Education, University and Research through the project AEROCOMP (contract MIUR no. DM48391), and thank Dr. Virgilio Brunetti, Center for Biomolecular Nanotechnologies @UNILE, Istituto Italiano di Tecnologia, Arnesano (LE), Italy, for his contribution to the confocal microscope study and Dr. Shilpi Karmakar, National Nanotechnology Laboratory (NNL), Institute Nanoscience - CNR, c/o Distretto Tecnologico, Lecce, Italy, for the help in interpreting the results.

${ }^{1}$ Z. Varga, G. Filipcsei, and M. Zrínyi, Polymer 46, 7779 (2005).

${ }^{2}$ J. Streque, A. Talbi, P. Pernod, and V. Preobrazhensky, IEEE Trans. Haptics 3, 88 (2010).

${ }^{3}$ J. Kim, S. E. Chung, S.-E. Choi, H. Lee, J. Kim, and S. Kwon, Nature Mater. 10, 747 (2011).

${ }^{4}$ F. Fahrni, M. W. J. Prins, and L. J. van IJzendoorn, J. Magn. Magn. Mater. 321, 1843 (2009). 
${ }^{5}$ M. Khoo and C. Liu, Sens. Actuators, A 89, 259 (2001).

${ }^{6}$ W. Wang, Z. Yao, J. C. Chen, and J. Fang, J. Micromech. Microeng. 14, 1321 (2004).

${ }^{7}$ F. N. Pirmoradi, J. K. Jackson, H. M. Burt, and M. Chiao, Lab Chip 11, 3072 (2011).

${ }^{8}$ F. Pirmoradi, L. Cheng, and M. Chiao, J. Micromech. Microeng. 20, 015032 (2010)

${ }^{9}$ J. Li, M. Z. L. Wang, W. Li, P. Sheng, and W. Wen, Microfluid. Nanofluid. 10, 919 (2011).

${ }^{10}$ G. Ausanio, C. L. Hison, V. Iannotti, L. Lanotte, and L. Lanotte, J. Appl. Phys. 110, 063903 (2011)

${ }^{11}$ Z. Varga, G. Filipcsei, and M. Zrínyi, Polymer 47, 227 (2006).

${ }^{12}$ R. M. Erb, R. Libanori, N. Rothfuchs, and A. R. Studart, Science 335, 199 (2012).

${ }^{13}$ J. Jestin, F. Cousin, I. Dubois, C. Ménager, R. Schweins, J. Oberdisse, and F. Boué, Adv. Mater. 20, 2533 (2008).

${ }^{14}$ A.-S. Robbes, F. Cousin, F. Meneau, F. Dalmas, F. Boué, and J. Jestin, Macromolecules 44, 8858 (2011).

${ }^{15}$ K. Keshoju and L. Sun, J. Appl. Phys. 105, 023515 (2009).

${ }^{16}$ D. Fragouli, R. Buonsanti, G. Bertoni, C. Sangregorio, C. Innocenti, A. Falqui, D. Gatteschi, P. D. Cozzoli, A. Athanassiou, and R. Cingolani, ACS Nano 4, 1873 (2010).

${ }^{17}$ H. Denver, T. Heiman, E. Martin, A. Gupta, and D.-A. Borca-Tasciuc, J. Appl. Phys. 106, 064909 (2009).

${ }^{18}$ W.-X. Fang, Z.-H. He, X.-Q. Xu, and H. Shen, Chin. Phys. Lett. 22, 2386 (2005).

${ }^{19}$ H. Fudouzi, Adv. Powder Technol. 20, 502 (2009).

${ }^{20}$ M. Jalali, S. Dauterstedt, A. Michaud, and R. Wuthrich, Composites, Part B 42, 1420 (2011).

${ }^{21}$ S. Y. Yang, H. E. Horng, C.-Y. Hong, H. C. Yang, M. C. Chou, C. T. Pan, and Y. H. Chao, J. Appl. Phys. 93, 3457 (2003).

${ }^{22}$ S. Y. Yang, Y. P. Chiu, B. Y. Jeang, H. E. Horng, C.-Y. Hong, and H. C. Yang, Appl. Phys. Lett. 79, 2372 (2001).

${ }^{23}$ Y. W. Huang, S. T. Hu, S. Y. Yang, H. E. Horng, J. C. Hung, C.-Y. Hong, H. C. Yang, C.-H. Chao, and C.-F. Lin, Opt. Lett. 29, 1867 (2004).

${ }^{24}$ G. A. Flores, J. Liu, M. Mohebi, and N. Jamasbi, Phys. Rev. E 59, 751 (1999).
${ }^{25}$ H. Wang, Y. Zhu, C. Boyd, W. Luo, A. Cebers, and R. E. Rosensweig, Phys Rev. Lett. 72, 1929 (1994).

${ }^{26}$ C.-Y. Hong, I. J. Jang, H. E. Horng, C. J. Hsu, Y. D. Yao, and H. C. Yang, J. Appl. Phys. 81, 4275 (1997).

${ }^{27}$ C.-Y. Hong, H. E. Horng, I. J. Jang, J. M. Wu, S. L. Lee, W. B. Yeung, and H. C. Yang, J. Appl. Phys. 83, 6771 (1998).

${ }^{28}$ H.-E. Horng, C.-Y. Hong, W. B. Yeung, and H.-C. Yang, Appl. Opt. 37, 2674 (1998).

${ }^{29}$ C.-H. Chang, C.-W. Tan, J. Miao, and G. Barbastathis, Nanotechnology 20, 495301 (2009).

${ }^{30}$ T. Hyeon, S. S. Lee, J. Park, Y. Chung, and H. B. Na, J. Am. Chem. Soc. 123, 12798 (2001).

${ }^{31}$ J. Park, E. Lee, N.-M. Hwang, M. Kang, S. C. Kim, Y. Hwang, J.-G. Park, H.-J. Noh, J.-Y. Kim, J.-H. Park, and T. Hyeon, Angew. Chem., Int. Ed. 44, 2872 (2005).

${ }^{32}$ P. Calcagnile, D. Fragouli, I. Bayer, G. C. Anyfantis, L. Martiradonna, D. Cozzoli, R. Cingolani, and A. Athanassiou, ACS Nano 6, 5413 (2012).

${ }^{33}$ W. Rasband, Image J 19972009 http://rsb.info.nih.gov/ij/, accessed February 18,2010

${ }^{34}$ V. Kalra, F. Escobedo, and Y. L. Joo, J. Chem. Phys. 132, 024901 (2010).

${ }^{35}$ J. Liu, E. M. Lawrence, A. Wu, M. L. Ivey, G. A. Flores, K. Javier, J. Bibette, and J. Richard, J. Phys. Rev. Lett. 74, 2828 (1995).

${ }^{36}$ J. Richardi, D. Ingert, and M. P. Pileni, Phys. Rev. E 66, 46306 (2002).

${ }^{37}$ F. M. Ytreberg and S. R. McKay, Phys. Rev. E 61, 4107 (2000).

${ }^{38}$ A. Satoh, J. Colloid Interface Sci. 150, 461 (1992).

${ }^{39}$ M. Respaud, J. Appl. Phys. 86, 556 (1999).

${ }^{40}$ G. Bertoni, B. Torre, A. Falqui, D. Fragouli, A. Athanassiou, and R. Cingolani, J. Phys. Chem. C 115, 7249 (2011).

${ }^{41}$ See supplementary material at http://dx.doi.org/10.1063/1.4759328 for angular dependence of the magnetic moment for Figure S1. Angular dependence of the magnetic moment for samples prepared using different values of B0 $(30,130$, and $280 \mathrm{mT})$. Figure S2. Results of NVT Monte Carlo simulations considering iron oxide NPs of diameter $\mathrm{d}=20 \mathrm{~nm}$ for the case of B0 $50 \mathrm{mT}$ (a) and $300 \mathrm{mT}$ (b). A video, acquired using the optical microscope, shows the kinetics of the formation of the magnetic chains in the elastomeric matrix upon the application of $\mathrm{B} 0=160 \mathrm{mT}$. 